\title{
Co-Ni Nanowires Arrays with Tunable Properties Obtained by Template Synthesis
}

\author{
Ruxandra Vidu',2, Andra Mihaela Predescu',*, Ecaterina Matei ${ }^{1}$, Andrei Berbecaru', Cristian Pantilimon', \\ Claudia Dragan', Mirela Sohaciu', Cristian Predescu' \\ ${ }^{1}$ University POLITEHNICA of Bucharest, Splaiul Independentei nr. 313, Bucharest, Sector 6, CP 060042, Romania \\ ${ }^{2}$ University of California Davis, Department of Electrical Engineering and Computer Sciences, One Shields Avenue, Davis, \\ CA 95616, USA
}

*Corresponding author: E-mail: andrapredescu@yahoo.com; Tel: (+40) 214029829

DOI: 10.5185 /amlett.2020.061524

Co-Ni nanowires arrays were obtained by electrochemical template synthesis in a polycarbonate track etched (PCTE) membrane. To diminish the effects of anomalous deposition observed in the Co-Ni system, the electrochemical deposition was performed from a solution containing nickel and cobalt in an atomic ratio of 3:1. Electrochemical deposition was performed at constant potentials $E=-0.8,-0.9$, $-1.0,-1.1,-1.2 \mathrm{~V}$ vs Ag/AgCl for $15 \mathrm{~min}$. The structures were characterized electrochemically via cyclic voltammetry, chronoamperometry, and charge stripping. Co-Ni nanowires were characterized by scanning electron microscopy (SEM/EDAX) to assess the morphology and the composition of the Co$\mathrm{Ni}$ alloy nanowires at different deposition potentials. Electrochemical and structural analysis provided details of their deposition kinetics, structure, and morphology, which would be used to build nanowires array with controlled structure and composition.

\section{Introduction}

Nanomaterials are integrated nowadays in all modern nano-devices. The reduced size helps electronics to have increased performance, low energy consumption and low heat output. Unique properties of nanomaterials are mainly related to the large surface to volume ratio but also to the 3D nano-architecture of the array, which can be used to trap the light by internal reflection when used in solar cells [1-4].

Compared to elemental metallic and semiconductor nanowires, the fabrication of compound or alloy nanowires imposes additional challenges. One technique to produce nanowires (NWs) is using template synthesis, in which the growth material is forced through the pores of a template. Electro-deposition in a template is a wellestablished technique for the inexpensive synthesis of nanowire arrays and has the advantages of low cost, ease of scalability, and highly tunable size control from diameters of a few nanometers to microns or larger. Any template with well-defined pores may be used, but tracketched polycarbonates (PCTE) or anodic aluminum oxide (AAO) are common due to their transversal nanopores. The technique may be used for the synthesis of nanowires, nanotubes, or intermediate structures, or even more complex geometries controlled by the choice of template and deposition protocol. Fabrication of nanowire array by template synthesis is presented in Fig. 1 for $\mathrm{Co}-\mathrm{Ni}$ alloy NWs. Briefly, the non-conductive membrane is coated with gold on one side of the membrane, which acts as electrode. Then, Co-Ni alloy is electro-chemically deposited from the Au base up through the nanopores. The nanowires array is exposed by dissolving the membrane in a solvent.

Iron-group alloys and nanomaterials fabricated therefrom are a broadly applicable class of materials for a wide range of applications, thanks to their conductivity and magnetic properties. Nanowires consisting of cobalt and nickel, in particular, hold promise for applications in magnetic media, miniaturized magnetic drivers, and MEMS. Also, NWs consisting of compounds containing nickel and cobalt find applications in batteries, thermoelectric and magnetic devices and sensing.

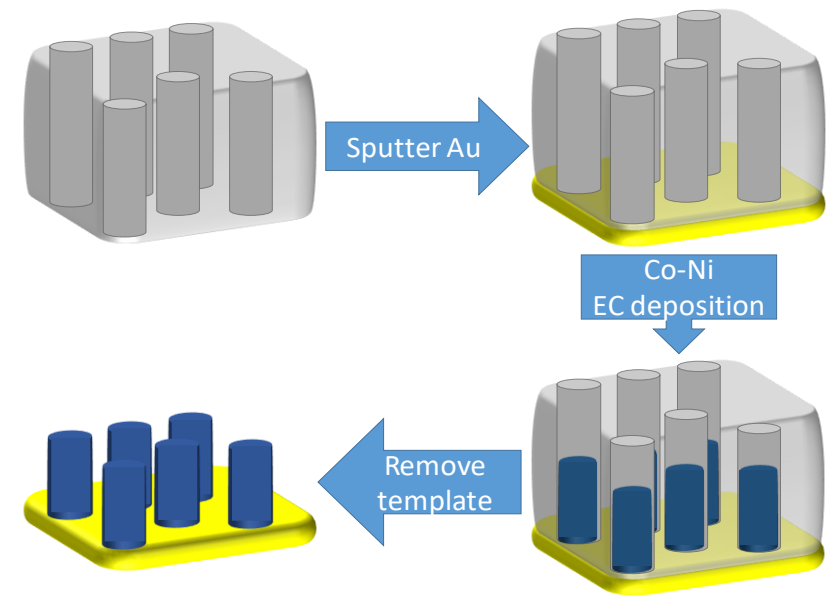

Fig. 1. Illustration of the template synthesis method to produce nanowire arrays.

Due to their ferromagnetic/nonmagnetic properties, Co-Ni nanowires can show either soft or hard magnetic behavior depending on the $\mathrm{Co} / \mathrm{Ni}$ ratio [5-7]. In order to 


\section{Advanced Materials Letters www. vbripress.com/aml}

obtain the nanowires with the magnetic behavior necessary to tailor the magnetic performance, nanowire array with controlled structure and composition should first be obtained. However, the iron-group alloys introduce additional challenges for the electrochemical deposition of this system due to the anomalous deposition [8-10].

Fig. 2 presents a cartoon of $\mathrm{Co}-\mathrm{Ni}$ anomalous electrochemical co-deposition, in which the electrochemically less noble Co deposits preferentially under most deposition potentials. In a normal electrodeposition, $\mathrm{Ni}$ and $\mathrm{Co}$ atoms deposit on the $\mathrm{Au}$ electrode in approximate proportion to their concentrations in solution. Simultaneously water is electrolyzed, forming $\mathrm{H}_{2}(\mathrm{~g})$, which escape, and $\mathrm{OH}^{-}$ions, which stay in solution and raise the local $\mathrm{pH}$. In anomalous deposition, hydroxide ions bond with metal ions to form $\mathrm{Co}(\mathrm{OH})^{+}$and $\mathrm{Ni}(\mathrm{OH})^{+}$. Because of the competitive adsorption, $\mathrm{Co}(\mathrm{OH})^{+}$adsorbs to the deposition sites more readily than the $\mathrm{Ni}(\mathrm{OH})^{+}$, effectively "crowding out" the Ni species and leading to the preferential deposition of Co over $\mathrm{Ni}$ [11].

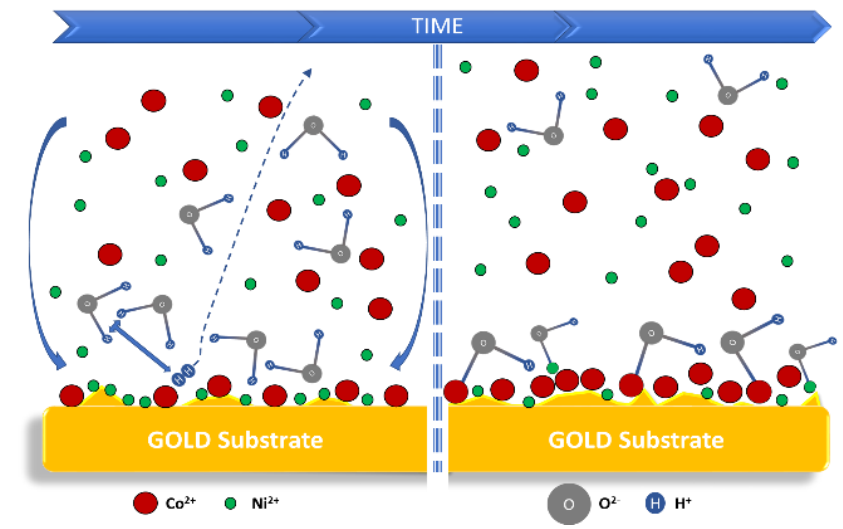

Fig. 2. Cartoon of normal $v s$ anomalous deposition. a) In normal electrodeposition, $\mathrm{Ni}$ (green) and Co (red) deposit on the Au electrode in approximate ratio to their potential; b) In anomalous deposition, Co atoms deposit preferentially over $\mathrm{Ni}$ on $\mathrm{Au}$.

Multi-segment nanowires of Co and Ni possess giant magnetostriction (GMS) and are thought for their magnetic properties. Presently, $\mathrm{Co}$ and $\mathrm{Ni}$ multi segmented nanowires are obtained from different growth baths, each segment being grown separately. Attempts have been done to grow multi-segmented nanowires of Co and Ni-rich alloy but anomalous phenomena prevented the deposition of high $\mathrm{Ni}$ concentration in the deposit by electrodeposition. In the present work, nanowires arrays of Co-Ni were grown by electrochemical template synthesis from citrate solutions containing $\mathrm{Co}$ and $\mathrm{Ni}$ ions in 1:3 ratio, in order to achieve a higher Ni concentration in the alloy. Nanowires were grown from solutions across the deposition potential range, and the effect of deposition potential on structure, morphology, and alloy composition was observed. These results provide the necessary information to construct $\mathrm{Co}$ and $\mathrm{Ni}$-rich multi-segmented nanowires with tailored magnetic properties for an array of possible applications in electronics, photonics, and other fields.

\section{Experimental}

\section{Materials/ chemicals details}

Solutions containing cobalt and nickel were prepared by dissolving $30 \mathrm{~g} / 1 \mathrm{NiSO}_{4} \cdot 6 \mathrm{H}_{2} \mathrm{O}$ and $10 \mathrm{~g} / \mathrm{l} \mathrm{CoSO} \mathrm{CoH}_{4} \cdot 6 \mathrm{H}_{2} \mathrm{O}$ in aqueous solutions containing $40 \mathrm{~g} / \mathrm{l} \mathrm{H}_{3} \mathrm{BO}_{3}$. All chemicals were purchased from Sigma-Aldrich. Deionized water (Milli Q 18-MX) was used for preparing solutions and for rinsing. Polycarbonate track-etched (PCTE, Sterlitech, Inc.) were used as templates.

\section{Material synthesis}

Sample electrodes were constructed from PCTE with an average pore size of $200 \mathrm{~nm}$ and thickness of $7 \mu \mathrm{m}$. Membranes were metallized with $\mathrm{Au}$ via sputter deposition to achieve an electrode with a thickness of approximately $50 \mathrm{~nm}$. A copper tape current collector with $\mathrm{Ni}$-free conductive adhesive was attached to the Au side of the membrane, and the membrane was encapsulated in plastic tape (Brother Corporation) except for a circular cut-out area of $0.3846 \mathrm{~cm}^{2}$ hole exposing the membrane surface. For film deposition the electrode was constructed with the $\mathrm{Au}$ electrode exposed to the solution; for nanowire deposition the non-coated side was exposed to solution, forcing deposition to occur within the pores. An illustration of the sample setup is shown elsewhere [1113].

Electrochemical experiments were performed in a conventional three-electrode setup including a potentiostat/ galvanostat PARSTAT 4000 (Princeton Applied Research AMETEK, USA). The counter electrode was made of $\mathrm{Pt}$ and the reference electrode was $\mathrm{Ag} / \mathrm{AgCl}$ electrode (3M $\mathrm{NaCl}$ ). The potentials presented here are relative to $\mathrm{Ag} / \mathrm{AgCl}(0.194 \mathrm{~V} v s$. SHE). All potentials given in this paper are given in reference to $\mathrm{Ag} / \mathrm{AgCl}$ unless otherwise specified. The Versa Studio software was used to control the computer. Deposition of Co-Ni NWs was performed at room temperature at constant potentials $\mathrm{E}=-0.8,-0.9,-1.0$, $-1.1,-1.2 \mathrm{~V}$ for $15 \mathrm{~min}$. Electrochemical characterization was performed by cyclic voltammetry, chronoamperometry, and charge stripping.

\section{Characterizations}

Structural and morphological characterization of nanowires was performed by scanning electron microscopy (SEM) equipped with an X-ray dispersive energy (EDS). To expose the nanowires array, the PCTE membrane was dissolved in a concentrated $\mathrm{Cl}_{2} \mathrm{CH}_{2}$ solution and then washed with Milli-Q water and dried with $\mathrm{N}_{2}$.

\section{Results and discussion}

\section{Electrochemical pre-treatment of the electrode}

An electrochemical treatment of the Au surface was performed in the beginning of each experiment in order to ensure same surface conditions before the electrodeposition experiment. The electrochemical 


\section{Advanced Materials Letters www. vbripress.com/aml}

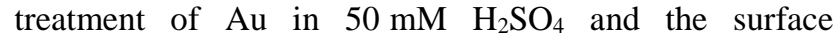
processes that led to a smooth $\mathrm{Au}$ surface are detailed elsewhere [14].

\section{Electrochemical characterization of the electrode in the growth solution}

Cyclic voltammetry (CV) experiments were performed from $0.7 \mathrm{~V}$ to a vertex potential of $-1.4 \mathrm{~V}$ at $20 \mathrm{mV} / \mathrm{s}$ (Fig. 3). Single-element deposition of $\mathrm{Co}$ and $\mathrm{Ni}$ are both well understood for films. The standard potential for the reduction half reaction of $\mathrm{Co}$ and $\mathrm{Ni}$ are very close to each other, i.e. -0.28 and $-0.25 \mathrm{~V}$ vs SHE, respectively [15]. The standard potential for co-deposition of $\mathrm{Co}$ and $\mathrm{Ni}$ is $0.53 \mathrm{~V}$ vs SHE (or $-0.485 \mathrm{~V}$ vs $\mathrm{Ag} / \mathrm{AgCl}$ ) which correspond to the potential at point $\mathrm{A}$ where an increase in current was observed in the $\mathrm{CV}$ scan going to the more negative potentials. Hydrogen evolution is overlapping to the co-deposition. The deposition of $\mathrm{Ni}$ and $\mathrm{Co}$ correspond to the desorption peaks $\mathrm{B}$ and $\mathrm{C}$ observed around 0.14 and $0.30 \mathrm{~V}$.

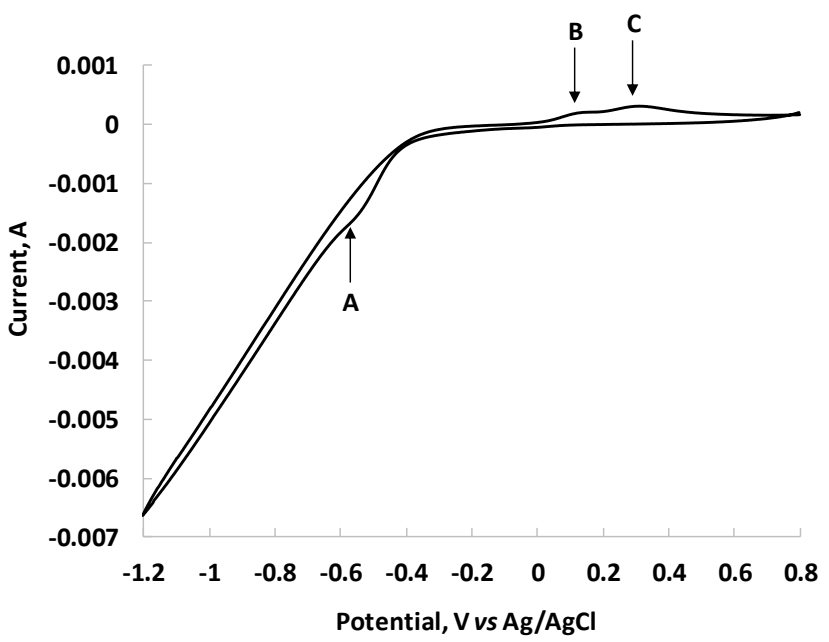

Fig. 3. Cyclic voltammetry of $\mathrm{Au}$ in the $\mathrm{Co}-\mathrm{Ni}$ solution at a sweep rate of $20 \mathrm{mV} / \mathrm{s}$.

\section{Electrochemical deposition}

Co-Ni deposition was performed in approximately $150 \mathrm{~mL}$ of solution for $15 \mathrm{~min}$ at room temperature. A typical deposition experiment ensued the following procedure: first, the open circuit potential (OCP) was measured, followed by two $\mathrm{CV}$ cycles from 0.7 to -1.2 and two $\mathrm{CV}$ cycles from -0.7 to $-1.4 \mathrm{~V}$, at a scan rate of $20 \mathrm{mV} / \mathrm{s}$. After cycling, the OCP was measured and then deposition was performed under a constant potential at -0.8, -0.9, -1.0, -1.1 and $-1.2 \mathrm{~V}$ vs $\mathrm{Ag} / \mathrm{AgCl}$ for 15 minutes, followed by OCP measurement.

Fig. 4 shows the variation of the deposition current in time at different deposition potentials. From $-0.8 \mathrm{~V}$ to -1.2 $\mathrm{V}$, the current moves to higher values and the hydrogen evolution becomes noticeable at potentials more negative than $-1 \mathrm{~V}$. Hydrogen deposition current on the Co-Ni alloy surface increases with increasing deposition current at higher overpotentials. Hydrogen evolution is a multi-step reaction process that inevitably occurs simultaneously with the electro-chemical deposition at negative potentials. The desorption of the adsorbed hydrogen, $\mathrm{H}_{\mathrm{ads}}$, is not seen in the $\mathrm{CV}$ due to the high surface diffusion of $\mathrm{H}_{\mathrm{ads}}$ and the formation of $\mathrm{H}_{2}$, which form bubbles and eventually evolve from the surface. In electro-chemical deposition, hydrogen evolution affects the morphology of the growth surface [16]. Gas bubbles formed on the surface have been visually observed at the membrane surface.

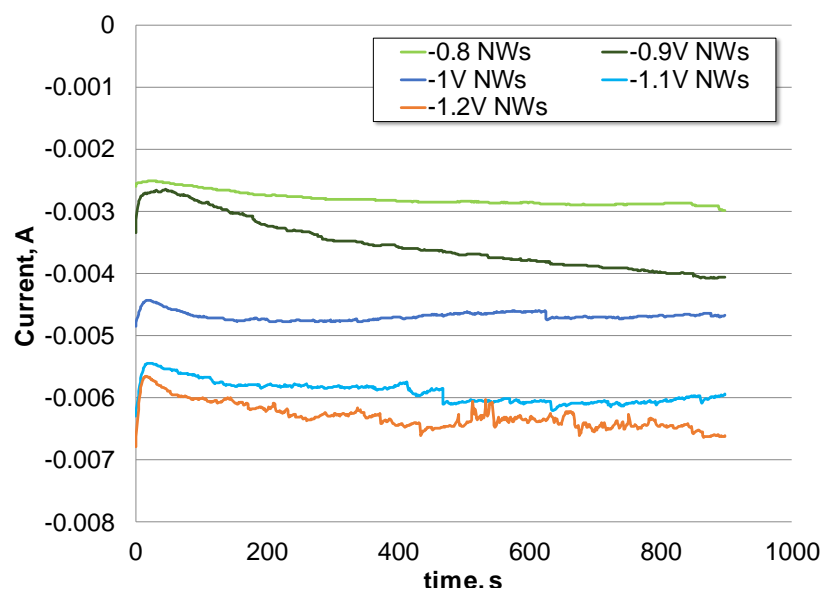

Fig. 4. The variation of deposition current in time.

\section{Chemical and morphological characterization}

SEM observation and EDAX were performed in order to correlate the composition of the deposit obtained at various deposition potential. From the SEM observation, we observed that the nanowire growth is very rapid. We were able to see nanowires only in the sample that were deposited at $-0.8 \mathrm{~V}$ (Fig. 5). All the other samples were covered with a layer that was characteristic to overgrown nanowires.
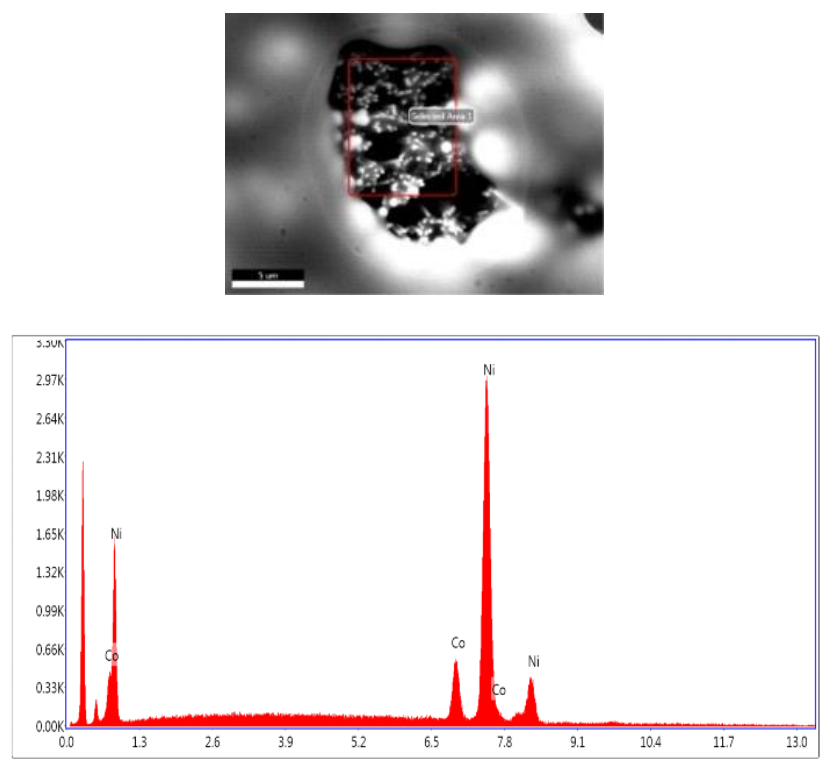

Fig. 5. EDS scan of the Co-Ni nanowires grown for $15 \mathrm{~min}$ at $-0.8 \mathrm{~V}$ (the inlet shows the SEM image of the area containing nanowires on which EDS measurement was performed. 


\section{Advanced Materials Letters www. vbripress.com/aml}

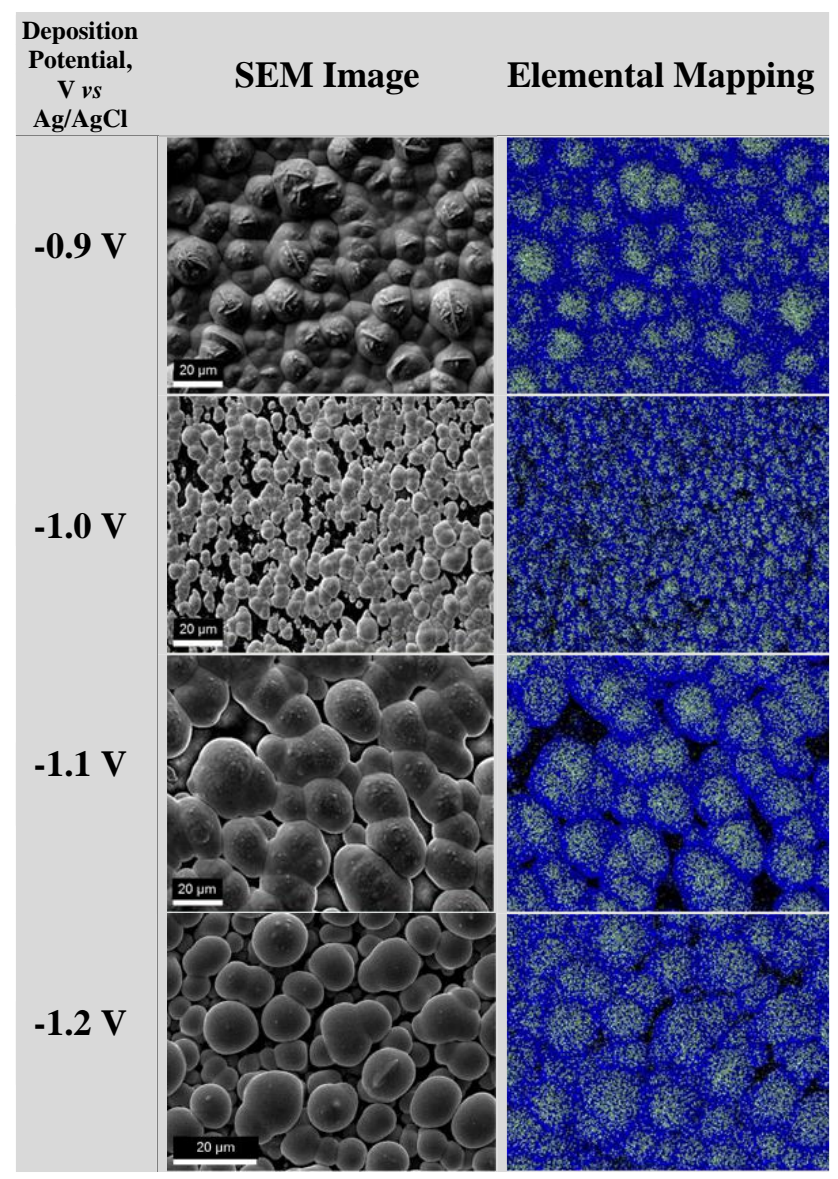

Fig. 6. SEM observation of the deposits obtained at potentials between 0.9 and $-1.2 \mathrm{~V}$.

Fig. 6 shows the surface morphologies along with the elemental mapping of the deposits obtained at potentials between -0.9 and $-1.2 \mathrm{~V}$. The morphology of the film obtained in the $-0.9 \sim-1.2 \mathrm{~V}$ potential range presents a similar network structure of mushroom caps, which is a feature of overgrown nanowires. The elemental mapping indicates that there is more $\mathrm{Co}$ than $\mathrm{Ni}$ on top of the mushroom caps than in between them. The film obtained at $-0.9 \mathrm{~V}$ shows also on top of the mushroom caps some features specific to Co deposition. The other films obtained at $-1,-1.1$ and $-1.2 \mathrm{~V}$ present also certain morphological features of Co-rich alloy deposition, which is consistent with the anomalous deposition theory.

The concentration of $\mathrm{Ni}$ in the deposit is shown in Fig. 7 along with the deposition rate, which was calculated from the charge deposited at each potential. The charge associated with the deposition at a certain potential, $Q(E)$, was estimated as the area under the curve, $i=f(t)$ (Fig. 4), according to the following equation:

$$
Q(E)=\sum_{t=1 s}^{t=900 s} i d t
$$

The increase in charge when the deposition potentials goes to more negative values doesn't show a linear behavior. It seems that the charge changes slope around $1.1 \mathrm{~V}$. Since the deposition rate also increases when overpotential increases, nanowires may grow faster at potentials more negative than $-1.1 \mathrm{~V}$. The overgrown of nanowires is associated with the formation of the so-called mushroom caps that continue to grow until they merge and cover the entire surface of the membrane with a continuous film.

The nanowires observed at $-0.8 \mathrm{~V}$ had $88 \% \mathrm{Ni}$ in the composition of the alloy. This value is the highest obtained until now. Prida et al. [17] obtained nanowires with $46 \% \mathrm{Ni}$ from a Watts type bath containing $0.36 \mathrm{M}$ $\mathrm{CoSO}_{4}, 0.04 \mathrm{M} \mathrm{CoCl}_{2}, 0.76 \mathrm{M} \mathrm{NiSO}_{4}, 0.13 \mathrm{M} \mathrm{NiCl}_{2}$, and $0.73 \mathrm{M} \mathrm{H}_{3} \mathrm{BO}_{3}$ in a gold-coated $\mathrm{H}$-AAO template. Based on this study, multi-segment nanowires were obtained, where the composition of each segment in the $\mathrm{Co}_{54} \mathrm{Ni}_{46}{ }^{-}$ $\mathrm{Co}_{85} \mathrm{Ni}_{15}$ nanowire was controlled by the applied pulse potential and time.

The composition of the Co-Ni alloy deposit obtained at potentials between -0.9 and $-1.2 \mathrm{~V}$ has between 73.89 and 77.03 at.\% Ni. However, the composition of the film is not uniform as the SEM/EDS images have shown (Fig. 6). Also, the hydrogen evolution interfered with the codeposition especially at more negative potentials than -0.8 V. Hydrogen evolution is much faster on a nanostructured surface such as the mushroom caps overgrown layer than on templated nanowire surface during growing.

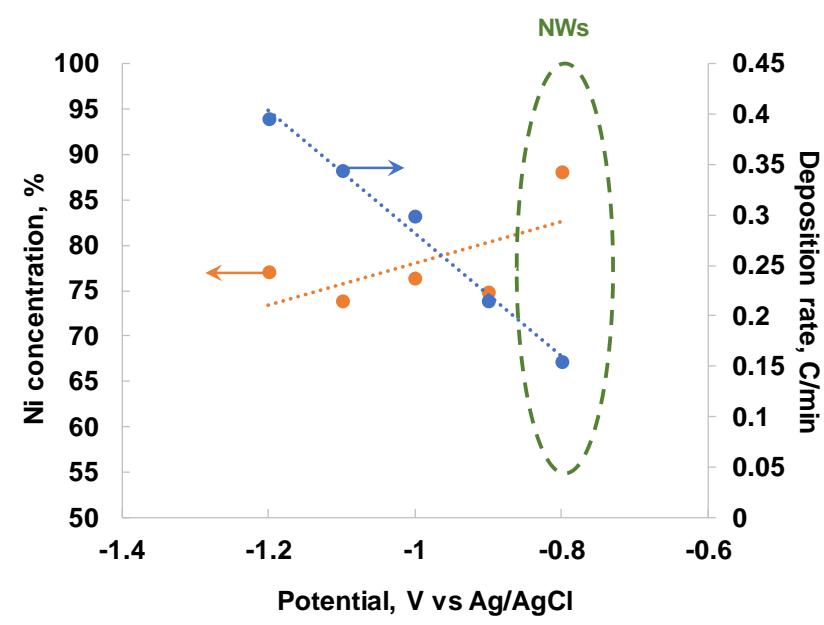

Fig. 7. Ni concentration in the Co-Ni deposit along with deposition rate at different deposition potentials.

Fig. 7 shows that $\mathrm{Ni}$ concentration in nanowires is $15 \%$ larger than $\mathrm{Ni}$ concentration in the outgrown mushroom caps. It is possible that Co deposition within the small pore size of the template delays its deposition. Previous data obtained for $\mathrm{Co}-\mathrm{Sb}[\mathbf{1 8}]$ have shown that there was a clear difference in composition between a thin film, nanowires and mushroom caps film. Also, the deposition of a film of a composition similar to nanowire takes place at more negative potential, and the mushroom caps formed by overgrown nanowire show a lower $\mathrm{Co}: \mathrm{Sb}$ ratio when compared to film or nanowires [18]. Anomalous deposition of Co-Ni alloys in pores differs from the films in large part because the growth environment inside a pore has no convection that keeps the solution near the interface in equilibrium with the bath. Phenomena that are sensitive to the diffusion of species 


\section{Advanced Materials Letters www. vbripress.com/aml}

may therefore show notable differences in a pore versus on a flat substrate, as seen in the unstable growth rate of nanowires within pores of differing lengths $[\mathbf{1 8}, \mathbf{1 9}]$.

\section{Conclusions}

Co-Ni NWs array have been obtained by electrochemical template synthesis in a solution containing $\mathrm{Co}: \mathrm{Ni}$ in a ratio of $1: 3$. In the electro-chemical deposition of $\mathrm{Co}-\mathrm{Ni}, \mathrm{Co}$ deposition is preferentially deposited over $\mathrm{Ni}$ due to the anomalous deposition shown by this system. Cobalt-nickel alloy forms a solid solution over the whole concentration range, which enables to modulate the magnetic properties according to the cobalt and nickel percentage. The electrochemical characterization of the deposition has shown that hydrogen evolution interferes with the growth of nanowires, but the effect was more pronounced on film produced by the overgrown nanowires, which formed mushroom caps when they reached the membrane surface. The overgrown nanowire film presented local nonuniform composition, i.e. the mushroom cups were rich in $\mathrm{Co}$ while the valleys in between them were rich in $\mathrm{Ni}$.

Co-Ni nanowires grown at $-0.8 \mathrm{~V}$ had a large $\mathrm{Ni}$ concentration of $88 \%$, the largest recorded for the $\mathrm{Co}-\mathrm{Ni}$ nanowires at this deposition potential. Co-Ni nanowires grown at potentials more negative than $-0.8 \mathrm{~V}$ presented overgrown morphologies such as mushroom cups. The deposition rate increases as the deposition potential moves to more negative values. However, the composition of the overgrown film does not vary much with the deposition potential. Ni concentration in the overgrown film was about $15 \%$ less that the nanowires obtained at $-0.8 \mathrm{~V}$.

Co-Ni alloy nanowires with tunable properties can be obtained by electrochemical template synthesis. By further optimizing the process, $\mathrm{Co}-\mathrm{Ni}$ alloy nanowire arrays can be incorporated in multiple applications. Further investigations of this system will be pursued to obtain multi-segmented nanowire arrays and study the magnetic properties of the array by varying the diameter of nanowires, the length and the composition of the segments.

\section{Acknowledgements}

The work has been funded by: POC Program, Project ID P_37_649.

\section{Author's contributions}

Conceived the plan: RV; Performed the expeirments: RV, AP, CPa, Data analysis: RV, AP, EM, AB, CPa, CD, MS, CP; Wrote the paper: RV. Authors have no competing financial interests.

\section{Keywords}

Ni-Co alloy, nanowires, anomalous deposition, template synthesis, electrochemical deposition.

Received: 16 August 2018

Revised: 14 January 2019

Accepted: 14 January 2019

\section{References}

1. Vidu, R.; Argo, B.P.; Stroeve, P.; Argo, J.; Islam S., Ku J.R.; Chen M., U.S. Patent 7847180.2010.

2. Vidu, R.; Argo, B.P.; Argo, J.; Stroeve, P.; Ku, J.R., U.S. Patent 8344241, 2013.

3. Ku, J.R.; Stroeve, P.; Vidu, R.; Talroze. R., U.S. patent Application 20060024438 A1, 2006.

4. Vidu, R.; Argo, B.P.; Argo, J.; Stroeve, P.; Ku J. R.; U.S. Patent $8877541,2014$.

5. Kikuchi, N., et al., IEEE Trans. Magn., 2001, 37, 4, 2082.

6. Sellmyer, D.J.; Zheng, M.; Skomski, R.; J. Phys.: Condens. Matter, 2001, 25, R433.

7. Mendez, M.; Gonzalez, S.; Vega, V.; Teixeira, J.M.; Hernando, B.; Luna, C.; Prida, V., Crystals, 2017, 7, 3, 66.

8. Gomez, E.; Ramirez, J.; Valles, E., J. Appl. Electrochem., 1998, 28, 1,71 .

9. Vazquez-Arenas, J.; Altamirano-Garcia, L.; Treeratanaphitak, T.; Pritzker, M.; Luna-Sanchez, R.; Cabrera-Sierra, R.; Electrochim. Acta, 2012, 65, 234.

10. Ignatova, K.; Marcheva, Y.; Bulg. Chem. Commun., 2015, 47, 2, 678.

11. Dryden, D.M.; Sun, T.; McCormick, R.; Hickey, R.; Vidu, R.; Stroeve, P.; Electrochim. Acta, 2016, 220, 595.

12. Dryden, D.M.; Vidu, R.; Stroeve, P.; Nanotechnology, 2016, 27, 445302.

13. Perez-Page, M.; Yu, E.; Li, J.; Rahman, M.; Dryden, D.M.; Vidu, R.; Stroeve, P.; Adv. Colloid Interface Sci., 2016, 234, 51.

14. Vidu, R., Perez-Page, M.; Quach, D.V.; Chen, X.Y.; Stroeve, P.; Electroanalysis, 2015, 27, 12, 2845.

15. CRC Handbook of Chemistry and Physics. 93rd Edition Ed. 20122013. 5-80. ISBN 9781439880494

16. Rojas, M., Fan, C. L.; Miao, H. J.; Piron, D. L.; J. Appl. Electrochem., 1992, 12, 1135.

17. Prida, V.M.; García, J.; Iglesias, L.; Vega, V.; Görlitz, D.; Nielsch C.; Barriga-Castro, E.D.; Mendoza-Reséndez, R.; Ponce, A; Luna C.; Nanoscale Res. Lett.; 2013, 8, 263.

18. Quach, D.V.; Vidu, R.; Groza, J.R.; Stroeve, P.; Ind. Eng. Chem. Res., 49, 2010, 22, 11385.

19. Vidu, R; Li, S.; Quach, D.V.; Stroeve, P.; J. Appl. Electrochem., 2012, 42, 5, 333. 